\title{
Evaluation des caractéristiques ethnobotaniques et structurales de Nesogordonia kabingaensis (K. Schum.) Capuron ex R. Germ. (Sterculiaceae) dans la forêt sacrée d'Ewè au Bénin en vue de la définition des stratégies de sa conservation
}

\author{
Hounnankpon YEDOMONHAN ${ }^{1 *}$, Aristide Cossi ADOMOU ${ }^{1}$, Marius AGUESSY ${ }^{2}$ et \\ François G. BOSSOU ${ }^{2}$
}

\author{
${ }^{I}$ Laboratoire de Botanique et Ecologie Végétale, Département de Biologie Végétale, \\ Faculté des Sciences et Techniques, Université d'Abomey-Calavi, 01 BP 4521 Cotonou, Bénin. \\ ${ }^{2}$ Direction Générale des Eaux et Forêts, 01 BP 4521 Cotonou, Bénin. \\ *Auteur correspondant; E-mail :h.yedo@yahoo.fr
}

\section{RESUME}

La dégradation des habitats naturels des espèces forestière évolue de façon croissance avec l'augmentation de la taille de la population du Bénin. L'objectif de cette étude est de contribuer à la conservation de Nesogordonia kabingaensis qui est l'une des espèces à habitat unique dans le pays. Des enquêtes ethnobotaniques auprès de 144 personnes ont été réalisées et les paramètres forestiers de $N$. kabingaensis ont été collectés dans 102 placeaux de $2500 \mathrm{~m}^{2}\left(50 \times 50 \mathrm{~m}^{2}\right)$ à l'intérieur de la forêt sacrée. Les enquêtés ont une bonne connaissance de l'espèce et $87,50 \%$ d'entre d'eux ont des critères spécifiques mais variés pour l'identifier. Au total, 14 types d'usages ont été recensés et les plus cités sont : les usages comme brosse végétale $(98,61 \%)$, la fabrication de mortier $(55,56 \%)$ et la confection de charpente de toit de maison (41,67\%). Les valeurs moyennes des paramètres dendrométriques sont de 30,64 cm pour le diamètre, $26,82 \mathrm{~m}$ pour la hauteur totale, $17,96 \mathrm{~m}$ pour la hauteur fût et de $5,40 \mathrm{~m}$ pour le diamètre de la voûte. Les paramètres structuraux sont en moyenne de 10 tiges/ha pour la densité et $0,76 \mathrm{~m}^{2} /$ ha pour la surface terrière. La régénération compte 399 tiges/ha. Les pressions humaines portent sur les individus jeunes et adultes qui sont exploités respectivement comme brosse végétale et bois d'œuvre/service. Elles sont si fortes qu'il est nécessaire de définir, à court terme, une stratégie de conservation de l'espèce in-situ et ex-situ. Ceci passera par l'introduction de $N$. kabingaensis dans les systèmes agroforestiers, son utilisation pour des plantations privées et le renforcement des mesures de protection de son habitat naturel.

(C) 2017 International Formulae Group. All rights reserved.

Mots clés : Nesogordonia kabingaensis, menace, savoir endogène, structure, conservation. 


\title{
Evaluation of ethnobotanical and structural characteristics of Nesogordonia kabingaensis (K. Schum.) Capuron ex R. Germ. (Sterculiaceae) in the sacred forest of Ewè in Benin in order to define strategies for its conservation
}

\begin{abstract}
The degradation of natural habitats of forest species is evolving in a growing way with the increase of the population size of Benin. The objective of this study is to contribute to the conservation of Nesogordonia kabingaensis which is one of the unique habitat species in the country. Ethnobotanical surveys of 144 people were carried out and the forest parameters of $N$. kabingaensis were collected in 102 plots of $2500 \mathrm{~m}^{2}(50 \times 50$ $\mathrm{m}^{2}$ ) within the sacred forest. The respondents have a good knowledge of the species and $87.50 \%$ of them have specific but varied criteria to identify it. In total, 14 types of use were identified and the most cited are: uses as a brush (98.61\%), mortar making (55.56\%) and the manufacture of roof framing (41.67\%). The average values of the dendrometric parameters are $30.64 \mathrm{~cm}$ for the diameter, $26.82 \mathrm{~m}$ for the total height, $17.96 \mathrm{~m}$ for the barrel height and $5.40 \mathrm{~m}$ for the diameter of the vault. The structural parameters are on average $10 \mathrm{stems} / \mathrm{ha}$ for the density and $0.76 \mathrm{~m}^{2} /$ ha for the basal area. Regeneration has $399 \mathrm{stems} / \mathrm{ha}$. Human pressures are on young and mature individuals who are exploited as brush and timber / service respectively. They are so strong that it is necessary to define, in the short term, a strategy for the conservation of in-situ and ex-situ species. This will involve the introduction of $N$. kabingaensis in agroforestry systems, its use for private plantations and the strengthening of protection measures for its natural habitat.

(C) 2017 International Formulae Group. All rights reserved.
\end{abstract}

Keywords: Nesogordonia kabingaensis, threat, indigenous knowledge, structure, conservation.

\section{INTRODUCTION}

Le Bénin est situé dans le couloir d'interruption de la forêt ouest-africaine, entrainant la descente de la savane jusqu'à la côte (Salzmann et Hoelzmann, 2005). Malgré la position défavorable de la végétation du Bénin dans le golfe de Guinée, les îlots rémanents de forêts denses humides semidécidues du pays révèlent d'une importance régionale et nationale. Au plan régional, ils présentent de grandes similarités floristiques (tant au niveau spécifique que des familles) avec ceux reconnus dans d'autres pays de l'Afrique de l'Ouest comme la Côte d'Ivoire et le Ghana où la forêt dense humide est moins perturbée en certains endroits (Adomou, 2005). Ensuite, au plan national, ces îlots de forêt comptent 517 espèces, soit $18,41 \%$ de la flore du Bénin (Akoègninou, 2004). De plus, 64\% des 106 espèces végétales menacées de disparition au Bénin sont inféodées à eux (Adomou et al., 2011). $\mathrm{Au}$ nombre des espèces menacées, certaines sont confinées à un seul îlot comme la forêt sacrée d'Ewè (Kétou) qui est le seul habitat du Bénin où sont localisées Mansonia altissima et Nesogordonia kabingaensis (Adomou, 2005). Cet auteur a classé ces 2 espèces comme en danger critique au Bénin. De plus, elles sont toutes inscrites sur la liste rouge de l'IUCN (2000) où M. altissima est aussi considérée comme en danger critique alors que $N$. kabingaensis est vulnérable. Un tel habitat abritant des espèces menacées à l'échelle mondiale devrait être alors une zone de forte priorité de conservation à cause de son unicité et des pressions anthropiques (Adomou, 2005). Malheureusement, la forêt sacrée d'Ewè est menacée de disparition à cause de l'absence d'une structure locale de gestion avec pour conséquence les coupes anarchiques de bois et l'empiètement dû à l'agriculture (Adomou, 2011). Si des données sur les degrés de menaces sont disponibles pour Mansonia altissima (Vitoekpon, 2011), elles sont par contre rares pour Nesogordonia kabingaensis.

En l'absence de mesure conservatoire, le Bénin risque la disparition de $N$. kabingaensis de son territoire étant donné que la croissance démographique galopante dont le taux actuel est de $3,5 \%$ (INSAE, 2013) ne 
fait qu'accentuer la pression anthropique sur les ressources forestières. Une telle disparition entraînerait non seulement la réduction de la biodiversité végétale du Bénin mais aussi la perte de tous les services (écologiques, sociales, culturelles et économiques) assurés par l'espèce. En effet, Nesogordonia kabingaensis, distribuée de la Sierra Leone au nord de la République Démocratique du Congo en Afrique de l'ouest, est une espèce faisant l'objet de commerce international comme bois d'œuvre et de service en Côte d'Ivoire, au Gabon et au Cameroun (Oyen, 2005). Elle constitue aussi une espèce agroforestière des cacaoyers en Côte d'Ivoire où elle est utilisée par les populations locales comme plante médicinale, bois de chauffe et bois d'œuvre (Adou Yao, 2016). Les divers travaux réalisés indiquent que les systèmes de connaissances sociales, culturelles et environnementales des communautés locales contribuent largement à la conservation de la biodiversité et à sa gestion pour le bien-être des populations (Takeuchi, 2010; Berkes, 2012 ; Hernandez-Morcillo et al., 2014).

L'objectif de cette étude est alors de répertorier les formes d'usages de l'espèce par les populations locales et d'évaluer la structure de $N$. kabingaensis au niveau de son habitat en vue de proposer des stratégies de sa conservation.

\section{MATERIEL ET METHODES Milieu d'étude}

La forêt sacrée d'Ewè, encore appelée Kouvizoun, appartient à l'arrondissement d'Adakplamè dans la Commune de Kétou (Figure 1). Sa superficie est de 737 ha 77 a 91 ca (MEHU, 2012). La Commune de Kétou à laquelle appartient le site d'étude est située dans la zone de climat subéquatorial (Akoègninou et al., 2006). La température moyenne mensuelle est de $27,1^{\circ} \mathrm{C}$ et varie de $25{ }^{\circ} \mathrm{C}$ en août à $29,1{ }^{\circ} \mathrm{C}$ en février. $\mathrm{La}$ pluviosité est en moyenne de 1191,5 mm d'eau pour la période de 1982 à 2013. Elle varie de $11 \mathrm{~mm}$ d'eau en décembre à $186 \mathrm{~mm}$ d'eau en mai. La moyenne annuelle de l'humidité relative de l'air est de $74 \%$. Sur le plan pédologique, deux types de sols sont rencontrés, à savoir: les sols ferralitiques faiblement désaturés et les sols ferrugineux tropicaux bien drainés. La végétation est une mosaïque de jachères, de champs, de plantations et de forêt dense humide semidécidue (Akoègninou et al., 2006). L'arrondissement d'Adakplamè compte 14.089 habitants, avec une densité de 88 habitants $/ \mathrm{km}^{2}$ (INSAE, 2013). Les ethnies dominantes sont les mahi et les nago. Les principales activités économiques des populations sont l'agriculture et la pêche. Outre ces 2 activités, les populations pratiquent les activités telles que l'exploitation forestière, l'élevage, la chasse et l'artisanat.

\section{Collecte de données}

La collecte de données a pris en compte aussi bien les connaissances endogènes des populations locales que les données biologiques sur l'espèce. En effet, ce sont ces populations qui sont au centre des interactions, des problèmes et des solutions à trouver (Roué et al., 2015). Par conséquent, les données ont été collectées par le biais d'enquêtes d'ethnobotaniques et de relevés forestiers.

Des enquêtes ethnobotaniques ont eu lieu par le biais d'interviews individuelles auprès de 144 personnes, choisies au hasard, dont 72 pour chacun des 2 villages (Adakplamè et Ewè) riverains à la forêt sacrée Kouvizoun. Les questions portaient sur le profil des enquêtés (âge, sexe, ethnie, profession, niveau d'instruction, religion), la parataxonomie de l'espèce (nom vernaculaire, critère de reconnaissance) et les usages (type d'usage, organe, mode et fréquence de prélèvement) de la plante. La répartition des enquêtés par catégories socioculturelles définies par Idohou et al. (2014) est donnée par le Tableau 1.

En ce qui concerne les relevés floristiques, 102 placeaux de $2500 \mathrm{~m}^{2}(50 \mathrm{~m} \mathrm{x}$ $50 \mathrm{~m}$ ) ont fait l'objet de relevés sur les 143 placeaux installés au hasard; les placeaux renfermant Nesogordonia kabingaensis étant les seuls considérés pour la collecte des données forestières sur l'espèce. Les données collectées sont les mesures dendrométriques et les données de régénération. Les données dendrométriques ont été prises pour tous les 
individus à $\mathrm{DBH}$ (diamètre à $1,30 \mathrm{~m}$ audessus du sol) $\geq 5 \mathrm{~cm}$. Elles sont : le DBH, la hauteur fût, la hauteur totale et le diamètre de voûte. Le diamètre de l'arbre et le diamètre de la voûte ont été mesurés respectivement avec un ruban $\pi$ et le décamètre; les hauteurs ont été prises avec un clinomètre. S'agissant de la régénération, tous les individus à $\mathrm{DBH}<5 \mathrm{~cm}$ ont été comptés. Ces paramètres sont ceux pris en compte pour l'étude structurale à l'échelle des communautés végétales et des espèces par divers auteurs dont Awokou et al. (2009), Adjonou et al. (2016a, 2016b), et Imani Mugisho et al. (2016).

\section{Analyse statistique}

Les données ethnobotaniques ont été analysées par des calculs de fréquences de réponses. Pour un paramètre donné, la fréquence de réponses est déterminée par le quotient, exprimé en pourcentage, du nombre de personnes ayant fourni la réponse par le nombre total d'enquêtés. L'analyse de variance (ANOVA) a permis de vérifier si le nombre d'usages cités par les enquêtés varie en fonction de leur catégorie socioculturelle. Le test de student a été utilisé pour comparer les moyennes. Le logiciel utilisé est MINITAB 14.

Les moyennes des paramètres dendrométriques (hauteur totale, hauteur fût et diamètres) et celles de la densité et de la surface terrière ont été calculées. La densité des arbres a été déterminée en nombre de tiges par hectare. La surface terrière $(\mathrm{G})$, exprimée en $\mathrm{m}^{2} /$ ha, a été calculée suivant la formule : $\mathrm{G}=\Sigma \pi(\mathrm{DBH})^{2} / 4$, où $\mathrm{DBH}$ est le diamètre à de la tige à $1,30 \mathrm{~m}$ au-dessus du sol.

Tableau 1 : Répartition de la taille des enquêtés au sein des catégories socioculturelles.

\begin{tabular}{lccc}
\hline \multirow{2}{*}{ Catégories socioculturelles } & Couches socioculturelles & $\begin{array}{c}\text { Taille de l'échantillon } \\
\text { d'enquêtés }\end{array}$ & $\begin{array}{c}\text { Pourcentage } \\
\text { d'enquêtés }\end{array}$ \\
\cline { 3 - 4 } Age (a) & Jeunes $(\mathrm{a}<30$ ans) & 32 & 22,22 \\
& adultes $(30 \leq \mathrm{a}<60$ ans) & 86 & 59,72 \\
Vieillards $(\geq 30$ ans) & 26 & 18,06 \\
\hline \multirow{2}{*}{ Ethnie } & Fon & 6 & 4,17 \\
& Mahi & 136 & 94,44 \\
Sexe & Nago & 2 & 1,39 \\
& Masculin & 88 & 61,11 \\
\multirow{3}{*}{ Profession } & Féminin & 56 & 38,89 \\
\hline \multirow{2}{*}{ Religion } & Agriculteur & 116 & 80,56 \\
& Commerçant & 6 & 4,17 \\
\hline \multirow{2}{*}{ Niveau d'instruction } & Artisan & 16 & 11,11 \\
& Guérisseur traditionnel & 6 & 4,17 \\
\hline & Chrétien & 62 & 43,06 \\
& Animiste & 82 & 56,94 \\
\hline
\end{tabular}




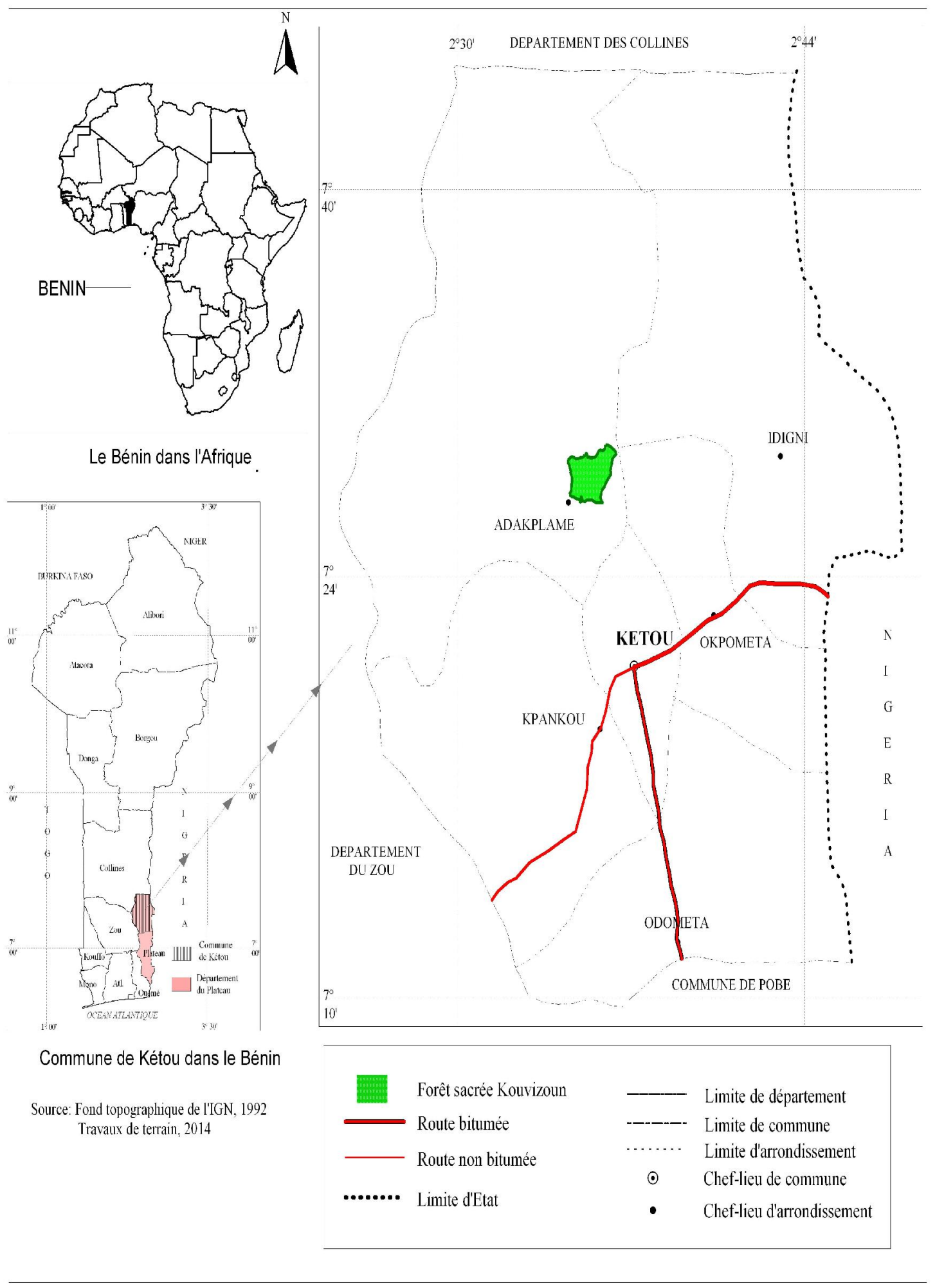

Figure 1 : Localisation géographique du milieu d'étude. 
RESULTATS

Caractéristiques ethnobotaniques de $N$. kabingaensis

\section{Parataxonomie de l'espèce}

Nesorgordonia kabingaensis est désignée par le nom "afaki" par 95,71\% des enquêtés. Elle est appelée par "afaki ahouvè", "afaki ahounvè" et "afaki ahouanvè" par $1,43 \%$ d'enquêtés pour chacune de ces appellations. Aucune de ces appellations n'est spécifique à un groupe ethnique.

Sur le plan de la reconnaissance de l'espèce, $87,50 \%$ des enquêtés ont des critères spécifiques de son identification mais les $12,50 \%$ restants n'arrivent pas à reconnaître la plante dans son habitat. Les critères de reconnaissance utilisés sont morphologiques et gustatifs. Il s'agit de : l'aspect de l'écorce (évoqué par $38,89 \%$ des personnes interviewées), l'aspect de la feuille $(23,61 \%)$, l'aspect de l'écorce et de la feuille $(20,83 \%)$ et le goût un peu amer de l'écorce $(4,17 \%)$.

Variabilité du nombre d'usages faits de l'espèce en fonction des couches et catégories socioprofessionnelles des enquêtés

Le nombre d'usages rapportés varie de 1 à 8 par enquêté, avec une moyenne de 3,24 $\pm 1,41$ (Tableau 2). Il présente des variations significatives d'une couche socioculturelle à l'autre au sein de trois catégories socioculturelles, à savoir le sexe $(\mathrm{P}=0,010 ; \mathrm{F}$ $=6,95 ;$ ddl. $=1)$, la profession $(\mathrm{P}=0,05 ; \mathrm{F}=$ $2,75$; ddl. $=3)$ et le niveau d'instruction $(\mathrm{P}=$ 0,$029 ; \mathrm{F}=3,74 ;$ ddl. $=2$ ). Pour les autres catégories socioculturelles (localité d'appartenance, âge, ethnie, religion) le nombre d'usages rapportés par enquêté n'induit pas de variation significative d'une couche socioculturelle à l'autre.

Les nombres moyens d'usages suivis de lettres différentes pour les couches d'une même catégorie socioculturelle sont significativement différents.

Au sein de 3 catégories socioculturelles des enquêtés, certaines couches ont une très forte contribution à la connaissance globale des divers usages de l'espèce au détriment d'autres. Ainsi, en prenant l'ethnie, les Mahi ont une connaissance totale des usages de $N$. kabingaensis alors que les Fon et Nago n'ont cité respectivement que 5 et 2 usages, soit donc 35,71 et $14,29 \%$ de contribution. De plus, en considérant la profession, les agriculteurs ont rapporté $92,86 \%$ des usages recensés dans la zone d'étude contre seulement $21,43 \%$ pour les commerçants. Les phytothérapeutes ont une contribution relativement importante de $64,19 \%$ alors qu'ils ne représentent que $4,17 \%$ des enquêtés. Enfin, tenant compte du niveau d'instruction, ce sont les illettrés qui ont la totalité de connaissances dans le domaine des usages de l'espèce. Les enquêtés instruits n'ont rapporté que $64,29 \%$ d'usages pour ceux du niveau primaire d'instruction et $28,57 \%$ pour le niveau secondaire.

Divers usages et formes d'exploitation de l'espèce

Les 14 usages recensés sont répartis dans 4 catégories dont les phytomédicaments (7 types d'usages), le bois d'œuvre et de service (5 types d'usages), l'hygiène des organes (1 type d'usages) puis le bois énergie (1 type d'usages) (Tableau 3). Les plus importants usages sont : l'utilisation de la tige comme brosse végétale pour l'hygiène buccodentaire (citée par 98,61\% des enquêtés), pour la fabrication de mortier $(55,56 \%)$ et pour l'édification des charpentes des toits de maison $(41,67 \%)$.

Les organes utilisés et leur mode d'acquisition varient en fonction des catégories d'usages. La tige est exploitée par l'abattage ou l'ébranchage des pieds vivants dans le cas des bois d'œuvre/service puis par ramassage des branches desséchées dans le cas des bois énergie.

En ce qui concerne l'utilisation de la plante comme brosse végétale, c'est aussi la tige qui est sollicitée et son acquisition se fait 
par 2 modes : la récolte et l'achat. La récolte dans la forêt est le mode le plus fréquent et est réalisé par $80,56 \%$ des personnes enquêtées. Par contre, l'achat aux marchés est pratiqué par 12,50\% de l'échantillon. Toutefois, 6,94\% des enquêtés font à la fois la récolte et l'achat. Pour la plupart des enquêtés, ce sont surtout les jeunes individus qui sont abattus pour leur exploitation comme brosse végétale. Ladite brosse végétale est utilisée quotidiennement les matins par $91,67 \%$ des enquêtés, de façon sporadique par 1,39\% de l'échantillon puis les matins et les soirs à la fois par 6,94\% de la population échantillonnée. Sur le plan de la fréquence d'usage, cette brosse végétale est utilisée tous les jours par 79,17\% des enquêtés et de façon sporadique par 5,56\%. Mais, $15,28 \%$ de l'échantillon ignorent la fréquence d'utilisation de l'espèce au cours d'une semaine.

Dans le cas du domaine de phytomédicaments, ce sont la tige, l'écorce de la tige et les feuilles qui sont les organes utilisés. L'écorce de la tige est utilisée dans le traitement de $71,42 \%$ des maladies citées et représente l'organe de $N$. kabingaensis le plus important en médecine traditionnelle. Les organes sont acquis par la récolte à l'intérieur de la forêt selon $100 \%$ des enquêtés.

\section{Caractéristiques forestières et structure démographique de Nesogordonia kabingaensis}

Les individus de $N$. kabingaensis recensés ont un diamètre moyen de $30,64 \mathrm{~cm}$, avec des arbres atteignant 75,41 cm (Tableau 4). La hauteur totale a une valeur maximale de $47,30 \mathrm{~m}$ et une moyenne de $26,82 \mathrm{~m}$. La hauteur fût est en moyenne de 17,96 m et les plus grands fûts ont $33,70 \mathrm{~m}$ de hauteur. Le diamètre de la voûte est compris entre 3,60 et $7,40 \mathrm{~m}$, avec une moyenne de 5,40 $\pm 0,92$.

La densité moyenne des arbres à $\mathrm{dbh} \geq$ $5 \mathrm{~cm}$ est de $10 \pm 6$ tiges/ha, avec une surface terrière de $0,76 \mathrm{~m}^{2} / \mathrm{ha}$.

La régénération naturelle a une densité moyenne de 399 tiges/ha avec un écart-type très élevé. Ceci traduit la grande variabilité de la régénération d'un placeau à l'autre.

La répartition de la densité par classe de diamètre des individus présente l'allure d'une courbe dissymétrique centrée sur la classe de diamètre de 20 à $30 \mathrm{~cm}$ qui compte $38 \%$ des arbres et arbustes (Figure 2). Cette classe de diamètre de 20 à $30 \mathrm{~cm}$ totalise, avec celle de 30 à $40 \mathrm{~cm}, 62 \%$ des effectifs des tiges. Ceci traduit une faible représentativité des individus de petit diamètre (moins de 20 $\mathrm{cm})$ qui n'ont qu'un taux de $16 \%$ et ceux de grand diamètre (plus de $40 \mathrm{~cm}$ ) ayant seulement une densité relative de $22 \%$. Cette distribution s'ajuste mieux à une fonction polynomiale dont l'équation est : $\mathrm{Y}=0,676 \mathrm{x}^{3}$ $-10,51 x^{2}+45,28 x-32,71\left(R^{2}=0,716\right)$.

La Figure 3 indique la répartition des individus par classe de hauteur. Elle présente aussi l'allure d'une courbe dissymétrique centrée sur la classe de 25 à $30 \mathrm{~m}$ de hauteur. Elle traduit alors la prédominance des individus ayant 25 à 30 de hauteur, avec un taux de 21,95\%. La distribution des individus par classe de hauteur s'ajuste mieux à une fonction polynomiale dont l'équation est : $\mathrm{Y}=$ $0,117 \mathrm{X}^{4}-2,052 \mathrm{X}^{3}+11 \mathrm{x}^{2}-19,46 \mathrm{x}+22,77$ $\left(\mathrm{R}^{2}=0,856\right)$. 
Tableau 2 : Nombre d'usages rapportés par catégorie et couche socioculturelles.

\begin{tabular}{llcc}
\hline $\begin{array}{l}\text { Catégories } \\
\text { socioculturelles }\end{array}$ & Couches socioculturelles & $\begin{array}{c}\text { Nombre moyen } \\
\text { d'usages rapportés } \\
\text { par enquêté }\end{array}$ & $\begin{array}{c}\text { Nombre total } \\
\text { d'usages } \\
\text { rapportés }\end{array}$ \\
\hline Localités & Adakplamè & $3,29 \pm 1,60^{\mathbf{a}}$ & 11 \\
d'appartenance & Ewè & $3,16 \pm 1,13^{\mathbf{a}}$ & 12 \\
\hline \multirow{2}{*}{ Age (a) } & Jeunes $(\mathrm{a}<30$ ans) & $3,00 \pm 1,41^{\mathbf{a}}$ & 8 \\
& adultes $(30 \leq \mathrm{a}<60$ ans) & $3,41 \pm 1,37^{\mathrm{a}}$ & 10 \\
\hline \multirow{2}{*}{ Ethnie } & Vieillards $(\geq 30$ ans) & $3,11 \pm 1,05^{\mathbf{a}}$ & 6 \\
& Fon & $3,67 \pm 1,56^{\mathbf{a}}$ & 5 \\
\multirow{2}{*}{ Sexe } & Mahi & $3,25 \pm 1,42^{\mathbf{a}}$ & 14 \\
& Nago & $2,00 \pm 0,00^{\mathbf{a}}$ & 2 \\
\hline \multirow{4}{*}{ Profession } & Féminin & $2,72 \pm 1,07^{\mathbf{a}}$ & 10 \\
& Masculin & $3,58 \pm 1,07^{\mathbf{b}}$ & 13 \\
\hline \multirow{2}{*}{ Religion } & Agriculture & $3,16 \pm 1,37^{\mathbf{a}}$ & 13 \\
& Artisanat & $3,63 \pm 0,91^{\mathbf{b}}$ & 7 \\
\hline \multirow{2}{*}{ Niveau d'instruction } & Commerce & $2,00 \pm 1,00^{\mathbf{c}}$ & 3 \\
& Phytothérapie & $5,00 \pm 2,65^{\mathbf{d}}$ & 10 \\
\hline Total & Animisme & $3,36 \pm 1,37^{\mathbf{a}}$ & 14 \\
\hline
\end{tabular}

Tableau 3 : Catégories et types d'usages avec leur fréquence de citation.

\begin{tabular}{lllc}
\hline Catégories & Types d'usages & Organes & $\begin{array}{c}\text { Fréquence de } \\
\text { citation }\end{array}$ \\
\hline Energie domestique & Bois énergie & tige & 50 \\
\hline \multirow{3}{*}{ Bois d'œuvre } & Charpente & tige & 41,67 \\
et de service & Porte et fenêtre & tige & 18,06 \\
& Manche de hache & tige & 9,72 \\
& Manche de houe & tige & 27,78 \\
& Mortier & tige & 55,56 \\
\hline Hygiène des organes & Brosse végétale & tige & 98,61 \\
\hline & Carie dentaire & tige & 8,33 \\
& Indigestion & écorce de tige & 1,39 \\
& Fièvre & écorce de tige & 4,17 \\
Phytomédicament & Fatigue infantile & écorce de tige & 1,39 \\
& Hypertension artérielle & écorce de tige & 1,39 \\
& Paludisme & feuilles & 1,39 \\
& Toux & écorce de tige & 5,56 \\
\hline
\end{tabular}


Tableau 4 : Valeurs moyennes des paramètres forestiers de $N$. kabingaensis

\begin{tabular}{lc}
\hline Paramètres forestiers & Moyenne et écart-type \\
\hline Diamètre $(\mathrm{cm})$ & $30,64 \pm 11,34$ \\
Hauteur totale $(\mathrm{m})$ & $26,82 \pm 9,31$ \\
Hauteur fût $(\mathrm{m})$ & $17,96 \pm 7,95$ \\
Diamètre de la voûte $(\mathrm{m})$ & $5,40 \pm 0,92$ \\
Densité (tiges $/ \mathrm{ha}$ ) & $10 \pm 6$ \\
Surface terrière moyenne $\left(\mathrm{m}^{2} / \mathrm{ha}\right)$ & $0,76 \pm 0,60$ \\
Densité de régénération $($ tiges $/ \mathrm{ha})$ & $399 \pm 436$ \\
\hline
\end{tabular}

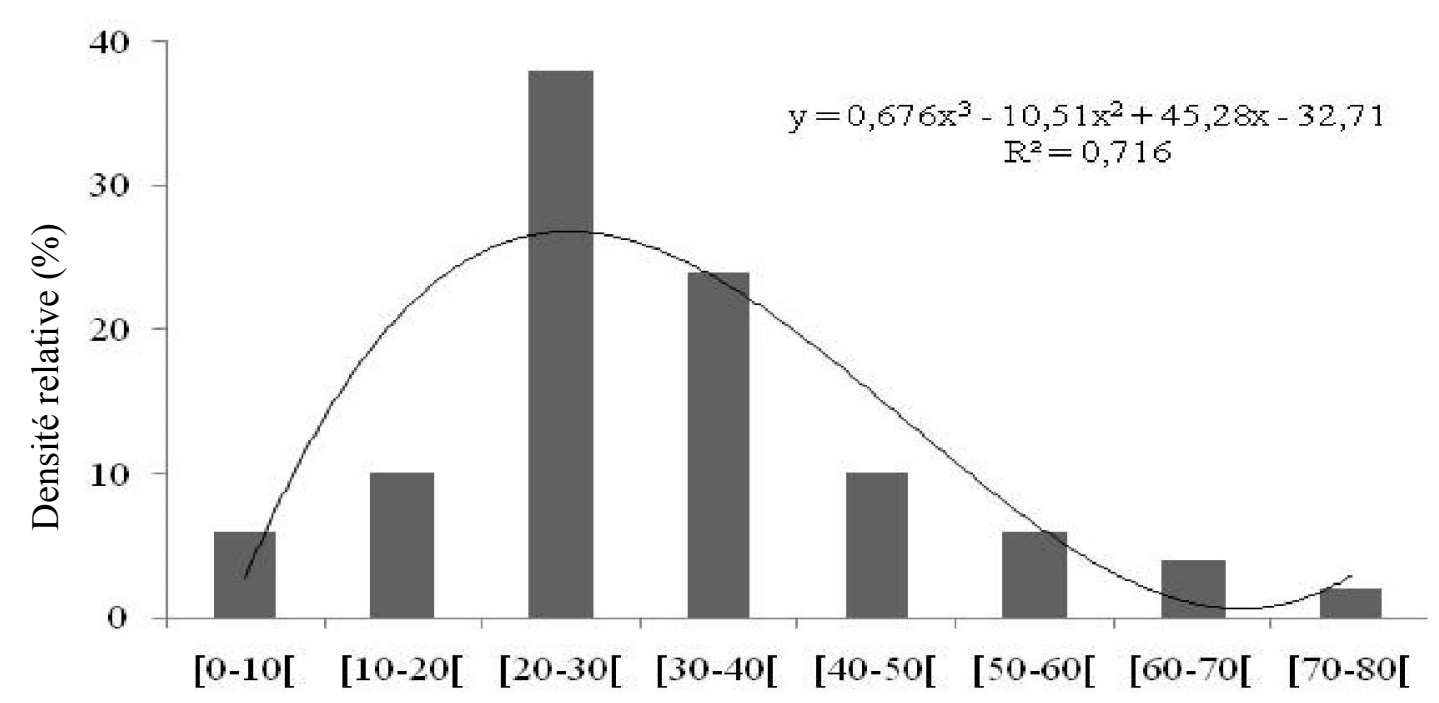

Classe de diamètre $(\mathrm{cm})$

Figure 2 : Répartition par classe de diamètre des individus de $N$. kabingaensis.

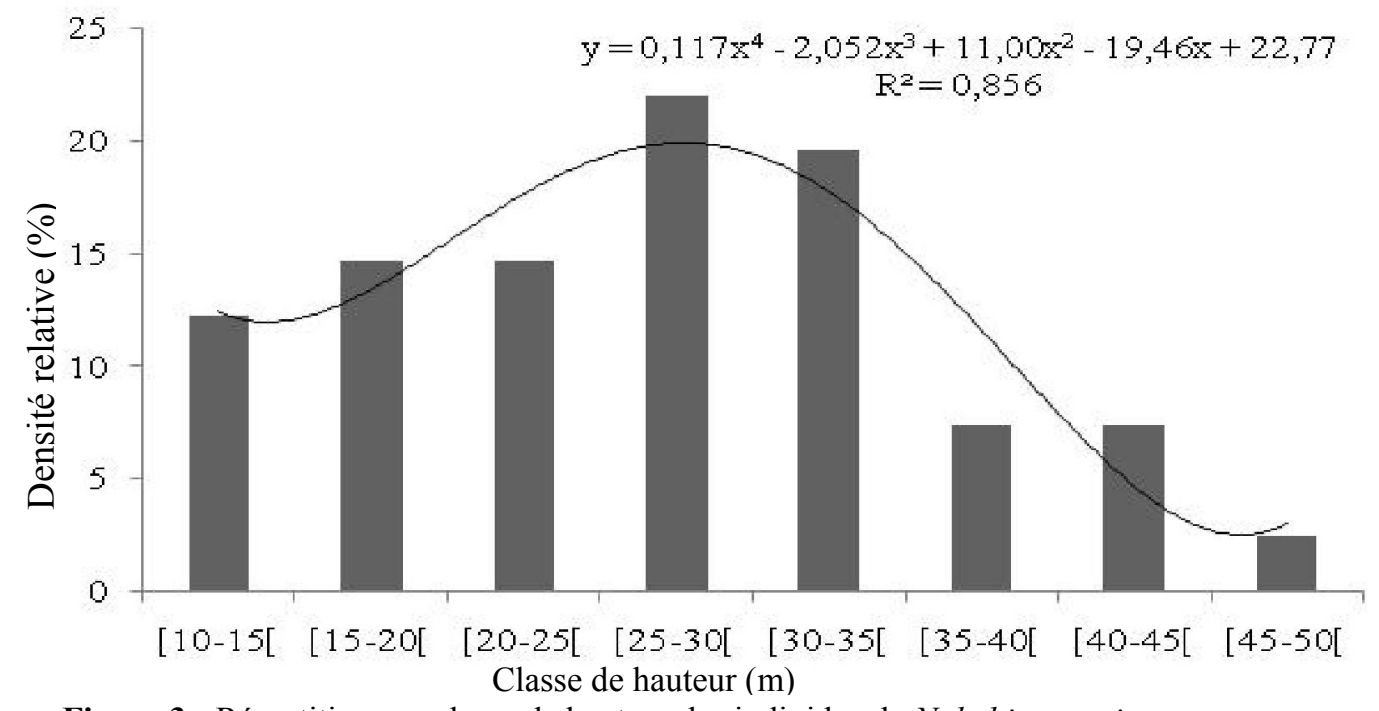

Figure 3 : Répartition par classe de hauteur des individus de $N$. kabingaensis. 


\section{DISCUSSION}

Degré de connaissance endogène des populations sur $N$. kabingaensis

L'étude parataxonomique a révélé l'unanimité de la population autour d'un nom vernaculaire pour Nesogordonia kabingaensis indépendamment des groupes linguistiques utilisateurs de l'espèce. L'utilisation de la même appellation de l'espèce par tous les 3 groupes linguistiques riverains à la forêt suppose que les 2 groupes linguistiques étrangers (Fon et Nago) à ces terroirs villageois auraient emprunté ce nom aux Mahi qui sont les autochtones (PADC, 2006). Les mots "ahouvè", "ahounvè" et ahouanvè", traduisant tous la couleur rouge en langues locales, ajoutés par certains enquêtés au nom populaire "afaki" semblent résulter des déformations phonétiques entrainant des écritures grammaticales différentes. Il s'agit là de mots descriptifs faisant allusion à la tranche rouge de son écorce et apportant donc un même qualificatif au nom "afaki" utilisé par tous. Ceci est réconforté par la grande proportion des enquêtés $(72,50 \%)$ qui font recours à l'aspect de l'écorce pour reconnaitre l'espèce dans son habitat. Ces résultats confirment les travaux de Spichiger et al. (2000) qui ont affirmé que l'utilisation des caractères botaniques utilitaires ou macroscopiques dans la nomenclature et l'identification des espèces ne date pas de l'ère des scientifiques mais de l'antiquité et continue, à nos jours, d'être appliquée par les communautés locales.

Sur le plan utilitaire, l'espèce est sollicitée dans 14 différents usages regroupés en 4 catégories. Ces résultats viennent enrichir la connaissance des différents types d'usages déjà connus de l'espèce. En effet, selon Oyen (2005), le bois de N. kabingaensis est fortement recherché comme bois d'œuvre/service à cause de sa dureté et de sa résistance à l'abrasion puis comme bois de chauffe dans de nombreux pays comme le
Cameroun, la Côte d'Ivoire, le Ghana et le Congo. De plus, Oyen (2005) a déjà indiqué que les rameaux de l'espèce sont aussi utilisés comme brosse végétale au Ghana et en Côte d'Ivoire et ses feuilles en décoction servent à soulager la carie dentaire chez le groupe ethnique Ando de la Côte d'Ivoire. Mais, par rapport à l'usage de la plante dans le traitement de la carie dentaire, les enquêtés de la présente étude ont notifié l'utilisation de la tige en mastication par opposition aux feuilles en décoction chez les Ando de la Côte d'Ivoire. Cette dissemblance dans l'organe utilisé pour le traitement de la carie dentaire n'est une particularité, ni de la plante, ni de la maladie. Elle est simplement liée au fait que la connaissance dans le domaine de la médecine traditionnelle varie d'une ethnie à l'autre comme l'a déjà souligné Rasingam et al. (2012). C'est ce qui explique que la présente étude a révélé, pour la première fois à nos connaissances, l'utilisation de $N$. kabingaensis dans le traitement de 6 autres maladies et symptômes (la toux, la fièvre, l'hypertension artérielle, le paludisme, la fatigue et l'indigestion). Ceci confirme d'autres travaux antérieurs (Philipps et al., 1994 ; Augereau, 2008 ; Ilumbe, 2010) pour lesquels une plante perçue comme d'une grande utilité par un groupe ethnique peut être considérée comme inutile par un autre.

Le nombre d'usages rapportés par personne a présenté des différences significatives en fonction du sexe, de la profession et du niveau d'instruction. Ceci met en évidence une connaissance inégale des enquêtés sur les usages de l'espèce. Cette distribution inégale des connaissances endogènes liées à l'utilisation de $N$. kabingaensis dans certaines couches sociales de la population a été déjà remarquée sur d'autres espèces végétales comme Blighia sapida (Ekué et al., 2010), Sclerocarya birrea (Gouwakinnou et al., 2011) et Chrysophyllum albidum (Gbesso, 2014). Ces résultats 
témoignent de ce que les usages sont plus détenus par les couches de la population qui restent au contact permanent des ressources phytogénétiques.

\section{Caractéristiques structurales du peuplement de $N$. kabingaensis}

Les individus matures de $N$. kabingaensis ont 45 à $50 \mathrm{~m}$ de hauteur et 80 à $120 \mathrm{~cm}$ de diamètre dans les forêts de la Côte d'Ivoire, du Ghana et de Congo (Oyen, 2005). La hauteur maximale de 47,3 $\mathrm{m}$ et le diamètre maximal de 75,41 cm obtenus dans le cadre de la présente étude sont très proches des valeurs optimales de croissance signalées. Ceci montre que les conditions climatiques et édaphiques du milieu d'étude conviennent bien au développement et à l'expansion de l'espèce.

La densité moyenne du peuplement de N. kabingaensis (10 tige/ha) s'intègre bien dans l'intervalle de 7-21 tiges/ha obtenues en Côte d'Ivoire pour la même espèce (Oyen, 2005). Elle est aussi similaire à la densité moyenne de 10-13 tiges/ha enregistrée pour Mansonia altissima (Vitoekpon, 2011) qui est une autre espèce menacée au Bénin et partageant le même habitat que $N$. kabingaensis (Adomou et al., 2011). La densité moyenne de régénération de $N$. kabingaensis qui est de 399 individus par hectare est similaire à celle de $M$. altissima (303 à 478 individus/ha) obtenue dans la même forêt par Vitoekpon (2011). La surface terrière obtenue $\left(0,76 \mathrm{~m}^{2} / \mathrm{ha}\right)$ est proche de celle de $0,96 \mathrm{~m}^{2} /$ ha signalée par Vitoekpon (2011) pour M. altissima dans la forêt sacrée Kouvizoun.

Les paramètres forestiers considérés au cours de cette étude (densité, surface terrière, taille moyenne des arbres et régénération) ont été retenus par Gehringer (2006) et Adjonou et al. (2016b) comme étant les bases techniques préliminaires pour la définition des objectifs d'aménagement et de conservation de la biodiversité. La densité des arbres de 10 tiges/ha obtenues et similaire à celles d'autres essences est témoin de ce que la forêt sacrée d'Ewé dispose encore d'un potentiel non négligeable d'individus de $N$. kabingaensis malgré la forte exploitation de cette espèce par les populations locales comme en témoignent les résultats d'enquêtes ethnobotaniques. L'allure dissymétrique que révèle la distribution par classe de diamètre des individus de $N$. kabingaensis traduit une pression humaine sélective sur les individus de petit et de gros diamètres. Ceci met en évidence la nécessité de prendre des mesures de conservation de l'espèce qui est d'un intérêt précieux pour les populations locales de part les divers services rendus par cette plante. L'une des mesures de conservation pourrait être l'intégration de $N$. kabingaensis dans les systèmes agroforestiers des agriculteurs et la réalisation des plantations privées. Il suffira donc d'appuyer ces communautés locales dans la production de jeunes plants de l'espèce à partir des pépinières villageoises. De plus, l'approche de l'agroforesterie nécessitera l'accompagnement des agriculteurs dans le choix des cultures à associer car la sélection des espèces agroforestiers résulte d'un compris entre leurs utilisations et leur incidence sur la culture (Sonwa et al., 2007). D'ailleurs, dans les systèmes agroforestiers, même des espèces indésirables sont maintenues à cause leurs usages (Adou Yao et al., 2016). La deuxième alternative de conservation de l'espèce est d'œuvrer pour renforcer la protection de son habitat en mettant en place une structure locale de gestion. La diminution des pressions humaines sur la forêt pourrait faciliter sa dynamique évolutive capable de générer, entre autres, une bonne structuration du peuplement de N. kabingaensis. En effet, la forêt sacrée d'Ewè dispose encore d'un potentiel de régénération assez important pour l'espèce. $\mathrm{Ce}$ potentiel de régénération qui est en 
moyenne de 399 plants/ha se situe bien dans l'intervalle de 176 à 876 plants/ha pour les 5 espèces les plus représentées en termes de régénération dans la Réserve de Faune de Togodo au sud du Togo (Adjonou et al., 2016b).

\section{Conclusion}

La présente étude qui a porté sur l'évaluation des caractéristiques ethnobotaniques et structurales de Nesogordonia kabingaensis dans la forêt sacrée d'Ewè dans l'arrondissement d'Adakplamè à Kétou a pour but de contribuer à la conservation de cette espèce qui est menacée de disparition au Bénin. Les résultats ethnobotaniques obtenus ont montré que l'espèce est fortement exploitée pour divers usages dont certains sont au quotidien. Le nombre d'usages connus par enquêté a montré une dépendance vis-à-vis de certaines couches sociales au sein des catégories socioculturelles des enquêtés. Les caractéristiques dendrométriques ont montré que les conditions écologiques du milieu sont adéquates et conviennent bien au développement de l'espèce comme les autres pays du bloc forestier ouest africain. Sur le plan structural, les paramètres évalués (répartition par classe de diamètre, densité, surface terrière, potentiel de régénération) ont montré que le peuplement de $N$. kabingaensis subit une forte pression humaine. Cette dernière affecte surtout les jeunes individus qui sont utilisés comme brosse végétale et les individus de gros diamètres qui servent de madrier dans la menuiserie.

La conservation durable de l'espèce nécessite la définition d'une stratégie appropriée de conservation intégrant aussi bien N. kabingaensis que son habitat. En effet, le risque de fragmentation de la forêt est assez grand au vue de sa très forte fréquentation humaine. Par ailleurs, il serait intéressant d'intégrer l'espèce dans les systèmes agroforestiers des populations d'une part et de promouvoir aussi sa plantation dans les champs et agglomérations d'autre part. A cet effet, l'assistance des agriculteurs sera nécessaire pour la réalisation des pépinières villageoises et la sélection des cultures à associer à la plante.

\section{CONFLIT D'INTERETS}

Les auteurs de cet article déclarent qu'il n'y a aucun conflit d'intérêts.

\section{CONTRIBUTIONS DES AUTEURS}

YH a assuré la conception de la méthodologie, la collecte et le traitement des données puis la rédaction du manuscrit. Les autres co-auteurs ont contribué à la collecte des données et à la relecture des différentes versions du manuscrit pour l'amélioration de sa qualité scientifique.

\section{REMERCIEMENTS}

Les auteurs remercient très sincèrement les populations riveraines de la forêt sacrée Kouvizoun pour leurs implications dans la collecte des données.

\section{REFERENCES}

Adjonou K, Djiwa O, Kombaté Y, Kokutse AD, Kokou K. 2016a. Etude de la dynamique spatiale et structure des forêts denses sèches reliques du Togo: implications pour une gestion durable des aires protégées. Int. J. Biol. Chem. Sci., 4(1) : 168-183.

Adjonou K, Radji AR, Kokutse AD, Kokou K. 2016b. Considération des caractéristiques structurales comme indicateurs écologiques d'aménagement forestier au Togo (Afrique de l'Ouest). VertigO, 16(1): 1-19.

Adomou CA. 2005. Vegetation patterns and environnemental gradients in Benin. Implications for biogeography and conservation. $\mathrm{PhD}$ Thesis. Wageningen 
University, Wageningen. $133 \mathrm{p}$.

Adomou AC, Agbani OP, Sinsin B. 2011.

Plantes Menacées de disparition au

Bénin. In Protection de la Nature en Afrique de l'Ouest : Une Liste Rouge pour le Bénin, Neuenschwander $\mathrm{P}$, Sinsin B, Georgen G (eds). IITA : Ibadan, Nigeria ; 21-46.

Adou Yao CY, Kpangui KB., Vroh BTA, Ouattara D. 2016. Pratiques culturales, valeurs d'usage et perception des paysans des espèces compagnes du cacaoyer dans des agroforêts traditionnelles au centre de la Côte d'Ivoire. Revue d'Ethnoécologie, 9: 119.

Akoègninou A. 2004. Recherches botaniques et écologiques sur les forêts actuelles du Bénin. Thèse d'Etat. Université de Cocody-Abidjan (Côte d'Ivoire), 326 p.

Akoègninou A, Van Der Burg WJ, Van Der Maesen LJG. 2006. Flore Analytique du Bénin. Backuys Publishers: Cotonou \&Wageningen ; 1034.

Augereau JM. 2008. Les plantes médicinales. In Aux Origines des Plantes, Hallé F, Lieutaghi P (eds). Fayard: Paris ; 170201.

Awokou KS, Ganglo CJ, Hessou A, Azontondé A, Adjakidjè V, de Foucault B. 2009. Caractéristiques structurales et écologiques des phytocénoses forestières de la forêt classée d'Itchèdè (Département du Plateau, Sud-est Bénin). Sciences et Nature, 6 : 125-138.

Berkes F. 2012. Sacred Ecology ( $3^{\text {rd }}$ edn). Routledge: New York, USA; 392.

Ekué MRM, Sinsin B, Eyog-Matig O, Finkeldey R. 2010. Uses, traditional, management, perception of variation and preferences in ackee (Blighia sapida K. D. Koenig) fruit traits in Benin: implications for domestication and conservation. J. Ethnobiol Ethnomed, 6: 1.
Gehring KR. 2006. Structure-based nonparametric target definition and assessment procedures with an application to riparian forest management. Forest Ecology and Management, 223: 125-138.

Gbesso GHF. 2014. Etudes écologique, ethnobotaniques et socio-économique de Chrysophyllum albidum G. Don (Sapotaceae) au Bénin. Thèse de Doctorat, Univ. Ab.-Cal., 168 p.

Gouwakinnou GN, Lykke AM, Assogbadjo AE, Sinsin B. 2011. Local knowledge, pattern and diversity of use of Sclerocarya birrea. J. Ethnobiol Ethnomed, 7: 8.

Hernandez-Morcillo M, Hoberg J, OterosRozas E, Plieninger T, GomezBaggethum E, Reyes-Garcia V. 2014. Traditional Ecology knowledge in Europe Status Quo and Insights for the Environmental Policy Agenda. Environment, 56: 3-17.

Idohou R, Fandohan B, Salako VK, Kassa B, Gbèdomon RC, Yédomonhan $\mathrm{H}$, Glèlè Kakaï RL, Assogbadjo AE. 2014. Biodiversity conservation in home gardens: traditional knowledge, use patterns and implications for management. International Journal of Biodiversity Science, Ecosystem Services \& Management, 10(2): 89-100.

Ilumbe G. 2010. Utilisation des plantes en médecine traditionnelle par les Pygmées (Ba-twa) et les Bantous (Ba-Oto) du territoire de Bikoro, province de l'Equateur en RD Congo. Thèse Doct. Univ. Libre de Bruxelles, 237 p.

Imani Mugisho G, Zapfack L, Bernard R, Mwanga MJC, Bulonwu F, Boyemba F. 2016. Variabilité structurale des peuplements d'arbres en forêt de Montagne du Parc National de KahuziBiega et ses environs, RD. Congo. European Scientific Journal, 12(23) : 88- 
111.

INSAE. 2013. Résultats provisoires du 4ème Recensement Général de la Population et de l'Habitat au Bénin. MDAEP, Bénin, 7 p.

IUCN (International Union for Conservation of Nature and Natural Resources). 2000. Red List of threatened species. Website: http://www.redlist.org.

MEHU. 2012. Projet d'Intégration des Forêts Sacrées dans le Système des Aires Protégées (PIFSAP): Potentiel en diversité biologique de 24 forêts sacrées des Départements de l'Ouémé et du Plateau au Bénin. Rapport, 304 p.

Oyen LPA. 2005. Nesogordonia kabingaensis (K.Schum.) Capuron ex R.Germ. In Prota 7(1): Timbers/Bois d'œuvre 1. [CD-Rom]. Louppe D, Oteng-Amoako AA, Brink M (eds). PROTA: Wageningen, Netherlands.

PADC. 2006. Monographie de la Commune de Kétou. Rapport Cabinet Afrique Conseil, 53 p.

Phillips OL, Gentry AH, Reynel C, Wilkin P, Ga'lvez-Durand BC. 1994. Quantitative ethnobotany and Amazonian conservation. Conservation Biology, 8: 225-248.

Rasingam L, Jeeva S, Kannan D. 2012. Dental care of Andaman and Nicobar folks: medicinal plants use as tooth stick. Asian Pacific Journal of Tropical Biomedicine, (2012): 1013-1016.

Roué M, Battesti V, Césard N, Simenel R. 2015. Ethnoecology of pollination and pollinators. Revue d'Ethnoécologie, 7,
DOI: 10.4000/ethnoecologie.2229.

Salzmann U, Hoelzmann P. 2005. The Dahomey Gap : an abrupt climatically induced rain forest fragmentation in West Africa during the late Holocene. The Holocene, 15(2): 1-10.

Sonwa DJ, Nkongmeneck BA, Weise SF, Tchatat M, Adesina AA, Janssens MJJ. 2007. Diversity of plants in cocoa agroforests in the humid forest zone of southern Cameroon. Biodiversity and Conservation, 16: 2385-2400.

Spichiger RE, Savolainen VV, Figeat M. 2000. Botanique Systématique des Plantes à fleurs: une Approche Phylogénétique Nouvelle des Angiospermes des Régions Tempérées et Tropicales. Presses Polytechniques et Universitaires Romandes: Italie; 373.

Takeuchi K. 2010. Rebuilding the relationship between people and nature: the satoyama initiative. Ecological Research, 25: 891-897.

Tossou MG. 2002. Recherche palynologique sur la vegetation Holocène du Sud-Bénin (Afrique de l'Ouest). Thèse de l'Université de Lomé, Option: Palynologie. Univ. de Lomé (Togo). 136 p.

Vitoekpon AI. 2011. Statut Statut de conservation de Mansonia altissima (A. Chev.) A. Chev. (bété) dans la forêt communautaire de Ewè-Adakplamè (commune de Kétou, au Bénin). Mém. Ing., $83 \mathrm{p}$. 